\title{
Editorial: Avoid the Tragedy of the Commons
}

In a recent Letter [1], Lin and Weitz noted that the tragedy of the commons [2] "occurs when individuals acting in their own self-interest deplete commonly held resources." Physical Review Letters (PRL) is a common resource of the global physics community, but its usefulness as a venue for the very best physics results would diminish if all the manuscripts authors wanted to publish were accepted. The history of PRL is marked by efforts to avoid the tragedy of the commons.

Already, near the end of PRL's third year, S. A. Goudsmit began an Editorial with "The Letters are getting out of hand again" [3]. His point was that too many published Letters would cause PRL to decline. Authors found publication in PRL to be valuable and wanted to publish more Letters, and the editors had to push back to maintain high quality.

The most recent changes of the review process and acceptance criteria were made in 2013 $[4,5]$. Now the number of published Letters is rising again. We editors will therefore once again renew our efforts to be selective, and again ask authors and reviewers to keep high standards in mind as you submit to and review for PRL. We note that there are many excellent journals in the Physical Review family that could be a venue for work that is not suitable for PRL. Please consider submission to those journals, to help PRL avoid the tragedy of the commons and remain a showcase for the most important work in all areas of physics.

Reinhardt B. Schuhmann

Published 12 June 2019

DOI: 10.1103/PhysRevLett.122.230001

[1] Y.-H. Lin and J. S. Weitz, Spatial Interactions and Oscillatory Tragedies of the Commons, Phys. Rev. Lett. 122, 148102 (2019).

[2] G. Hardin, The Tragedy of the Commons, Science 162, 1243 (1968).
[3] S. A. Goudsmit, Editorial, Phys. Rev. Lett. 6, 587 (1961).

[4] P. Meystre, Editorial: Meystre Presents Plans to Maintain and Strengthen PRL as the Premier Physics Journal, Phys. Rev. Lett. 111, 100002 (2013).

[5] P. Meystre, Editorial: Review Changes, Phys. Rev. Lett. 111, 180001 (2013). 